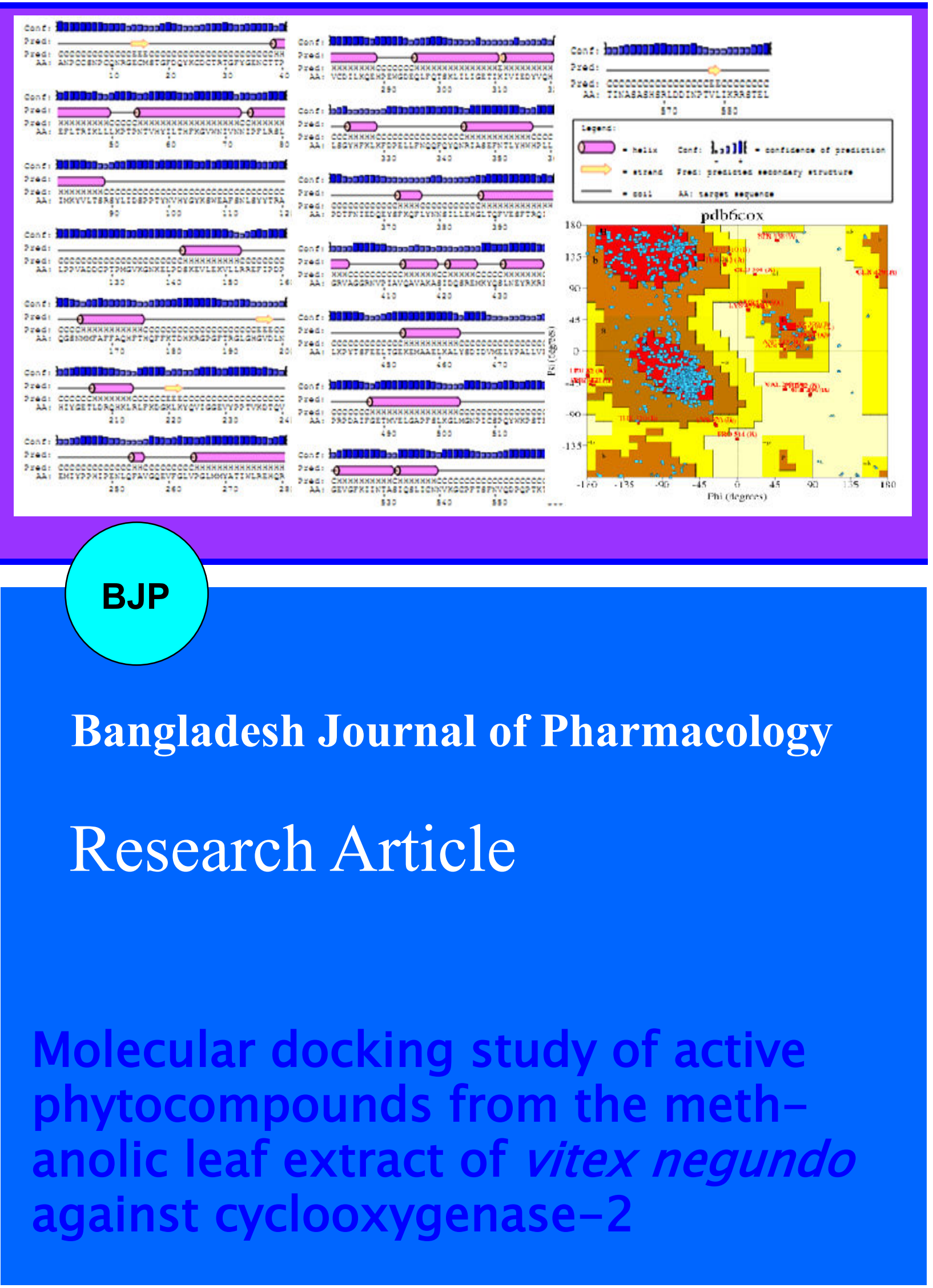




\title{
Molecular docking study of active phytocompounds from the methanolic leaf extract of vitex negundo against cyclooxygenase-2
}

\author{
Deepa Murugesan, Renuka Devi Ponnuswamy and Dhanalakshmi Karur Gopalan
}

Department of Biotechnology, Anna University, Regional centre, Coimbatore, Tamil Nadu 640 047, India.

\begin{tabular}{|c|c|}
\hline \multicolumn{2}{|l|}{ Article Info } \\
\hline Received: & 5 January 2014 \\
\hline Accepted: & 30 January 2014 \\
\hline Available Online: & 29 March 2014 \\
\hline \multicolumn{2}{|c|}{ DOI: 10.3329/bjp.v9i2.17543 } \\
\hline \multicolumn{2}{|c|}{$\begin{array}{l}\text { Cite this article: } \\
\text { Murugesan D, Ponnuswamy RD, } \\
\text { Gopalan DK. Molecular docking } \\
\text { study of active phytocompounds } \\
\text { from the methanolic leaf extract of } \\
\text { vitex negundo against cyclooxygenase- } \\
\text { 2. Bangladesh J Pharmacol. 2014; 9: } \\
\text { 146-53. }\end{array}$} \\
\hline
\end{tabular}

\begin{abstract}
The aim of the study is to identify the phytocompounds with antiinflammatory properties from the methanolic leaf extract of Vitex negundo and also to find the inhibitors of cyclooxygenase-2 (COX-2) enzyme through molecular docking. GC-MS was performed for the methanolic leaf extract of $V$. negundo. Various phenolic phytocompounds were identified through GCMS. This Study has illustrated the binding of four biologically active compounds from the methanolic extract of $V$. negundo against the inflammation associated target COX-2 enzymes. The binding energy is evaluated through docking studies of the ligand with the target protein 6COX_A. These Phytochemical compounds have a good docking score and glide energy. Based on the results, binding energy was compared with the known COX-2 inhibitory compounds namely aspirin and ibuprofen. It is understood that these phytochemical compounds can be considered as strong inhibitors for COX-2 and possess potential medicinal values with antiinflammatory properties.
\end{abstract}

\section{Introduction}

Herbal medicines are of great importance in primary healthcare of individuals and communities in many developing countries (Ghosh, 2003). Plants are good source of biologically active compounds. In recent years, plant products play a dominant role in the discovery of phytodrugs for treating human diseases. People show their interest towards natural compounds from plant materials for treatment of various ailments. There is a worldwide interest in searching for safe and new phytochemical drugs. Inflammation is an important physiological reaction which occurs in response to a wide variety of injurious agents (e.g. bacterial infection, physical trauma, chemicals or any other phenomenon) ultimately aiming to perform the dual function of limiting damage and promoting tissue repair (Nathan, 2002).

The COX enzyme exists in two isoforms (COX-1 and
COX-2). The COX-1 enzyme protects the stomach lining from corrosive acids and digestive chemicals. It also helps to maintain kidney function.

COX-1 and COX-2 are $63 \%$ identical and $77 \%$ similar at the amino acid level. The major differences are at the Nterminal and C-terminal regions (Gierse et al., 1996). COX-2 is an oxidoreductase enzyme playing a role in prostaglandin biosynthesis and inflammatory responses. The COX-2 enzyme binds to arachidonic acid which causes pain and inflammation (Sudha et al., 2008). COX2 is one of the well-known targets for the antiinflammatory therapy. Inhibition of COX-2 can provide relief from the symptoms of inflammation and pain (Saqib, 2009). Non-steroidal anti-inflammatory drugs (NSAIDs) influence their effects by inhibiting prostaglandin production (Kurumbail et al., 1996). COX-2 is an important protein which attracts the researchers for their study in the past few decades. The sequence and structural studies reveal Mus musculus COX-2 shares 
the common conserved sequence and structural pattern with human COX-2.

Vitex negundo Linn (Verbenaceae) is commonly known as five-leaved chaste tree or Monk's pepper. It grows in waste lands and is also planted as a hedge plant. All parts of $V$. negundo are used as medicine in the indigenous system of medicine. All parts of the plant are used for treatment of a wide spectrum of health disorders in traditional and folk medicine (Vishwanathan and Basavaraju, 2010). V. negundo have certain bioactive molecules and many of the drugs are based on these bio active molecules. $V$. negundo is one of the medicinal plants used in traditional medicine and reported to have many pharmacological activities such as anti-inflammatory activity (Telang et al., 1999; Dharmasiri et al., 2003; Kulkarni et al., 2008), antioxidant activity (Tiwari and Tripathi, 2007), antibacterial activity (Aswar et al., 2009 ; Khokra et al., 2008) and antifungal activity (Mahmud et al., 2009). The leaves are the most potent part of the plant for medicinal purposes. It is used for treatment of eye disease, toothache, enlargement of the spleen, rheumatoid arthritis, gonorrhoea, and bronchitis (Tandon, 2005; Raji, 2013) .

\section{Methods and Materials \\ Collection of plant material}

The fresh mature plant leaves of $V$. negundo were collected from Maruthamalai hill area in Coimbatore district in the month of July 2011. The collected plant materials were identified authentically by Dr. G.V. Murthy. Scientist ' $F$ ' from Botanical Survey of India, Southern Circle Coimbatore, Tamil Nadu, India.

\section{Preparation of extract}

The mature fresh leaves of $V$. negundo were washed in fresh water thoroughly 2-3 times and finally with sterile water. The leaves were dried on sterile blotter under shade and then powdered in a mixture grinder. $150 \mathrm{~g}$ of shade dried powder was cold macentrated with $500 \mathrm{~mL}$ of Methanol for 48 hours and then filtered. The solvent was then evaporated at a constant temperature of $40^{\circ} \mathrm{C}$ until a concentrated extract was obtained. Crude extract obtained were kept at $4^{\circ} \mathrm{C}$ until further assay.

\section{Preparation of extract}

One $\mu \mathrm{L}$ of the Methanol leaf extract of $V$. negundo was employed for GC/MS analysis.

\section{Instruments and chromatographic conditions}

GC-MS analysis was carried out on a DB 5 - MS capillary standard non - polar column and gas chromatograph interfaced to a mass spectrometer (GCMS) instrument employing the following conditions: column elite- 1 fused silica capillary column $(30 \times 0.25$ mm i.d. $\times 1$ EM df, composed of $100 \%$ dimethyl polysiloxane), operating in electron impact mode at 70 $\mathrm{eV}$; helium $(99.9 \%)$ was used as carrier gas at a constant flow of $1 \mathrm{~mL} / \mathrm{min}$. The oven temperature was programmed from $80^{\circ} \mathrm{C}$ (isothermal for $2 \mathrm{~min}$ ), with an increase of 8 to $250^{\circ} \mathrm{C} / \mathrm{min}$. Mass spectra was taken at $70 \mathrm{eV}$; a scan interval of $0.5 \mathrm{~s}$ and fragments from 40 to $550 \mathrm{Da}$.

\section{Identification of phytochemical compound}

The mass spectrums of the unknown components were compared with the spectrum of the known components stored in the Wiley9 library. The name, molecular weight and structure of the components of the test materials were ascertained.

\section{Molecular docking studies}

Molecular docking studies have been carried out by using GLIDE (Grid-based Ligand Docking with Energetics) software v5.5 developed by Schrödinger running on Red Hat Enterprise Linux 5 (RHEL5) workstation. Maestro v9.0 Graphical User Interface (GUI) workspace was used for all the steps involved in ligand preparation, protein preparation and Induced Fit Docking (IFD).

\section{Ligand preparation}

The structures of the phytocompounds obtained from the result of GCMS, Wiley9 library and the structures were drawn by using Chem Sketch (ACDLABS 12.0) and converted into 3D structure with help of a 3D optimization tool of ACD Labs Software and the structures were saved in the MOL format. The structures of aspirin and ibuprofen were obtained from PubChem. The ligands used in this study were prepared using LigPrep (Castro et al., 1968) module of v2.3 of Schrödinger Suite, 2009. Using the Impact module of glide the ligands were minimized with 1000 cycles of steepest gradient and 5000 cycles of conjugate gradient.

\section{Retrieval of target sequence}

The FASTA format of Mus musculus COX-2 with Accession number 6COX_A was retrieved from the NCBI protein database. The protein sequence contains 587 amino acid residues.

\section{COX-2 enzyme protein structure}

The target X-ray crystal structure of the COX-2 enzyme protein having the resolution of $2.8 \mathrm{~A}^{\circ}$ was retrieved from Protein Data Bank (PDB) with ID 6COX_A. The 6COX_A is a complex of COX-2 with an inhibitor SC558 (http://www.rcsb.org/pdb).

\section{Secondary structure prediction}

The secondary structure of 6COX_A was predicted using PSIPRED v3.0 (Bryson et al., 2005; Jones, 1999). 
Neural nets are used to convert PsiBlast profile data to secondary structure propensities. A putative secondary structure is obtained for each residue associated with a confidence value for the prediction

\section{Structure validation}

The PDB protein structure was evaluated using the ProQ web server (Wallner, 2003). The models were validated using PROCHECK program (Laskowski et al., 1993).

\section{Active site analysis}

The receptor accessible pockets and active sites of COX2 were identified by computed atlas of surface topography of proteins (CASTp) calculation (Dundas et al., 2006). The accessibility of the pockets were tested by docking with phytochemical inhibitor molecules.

\section{Preparation of 6COX_A protein}

The X-ray crystal structure of COX-2 (PDB id: 6COX_A) retrieved from $\mathrm{PDB}$ is a monomeric structure. It consists of 287 amino acids along with an inhibitor SC - 558 molecule. The raw PDB protein structure could not be used for molecular docking studies. PDB structure consists only of heavy atoms, waters, cofactors, metal ions and can be of multimeric. These structures do not have the information about bond orders, topologies or formal atomic charges. The terminal amide groups may be misaligned because the $\mathrm{X}$-ray structure analysis cannot distinguish between $\mathrm{O}$ and $\mathrm{NH}_{2}$ Ionization and tautomeric states are also unassigned. So, the raw PDB structure retrieved from PDB should be prepared in a suitable manner for docking. Protein Preparation Wizard of GLIDE software was used to process and prepare the protein. This Wizard allows one to properly prepare a protein for docking. This also follows the Optimized Potential for Liquid Simulations. All Atoms (OPLS-AA) force fields for energy minimization. The X-ray crystal structure of 6COX_A protein was prepared by removing all the water molecules present in the structure. Since the raw data do not contain any hydrogen in it, the implicit hydrogen atoms were added to the atoms to satisfy their appropriate valencies. Then the structure was optimized by assigning the bond orders, bond angles and topology. The formal atomic charges were fixed for the amino acid residues. The optimized structure was then energy minimized to remove the steric clashes between the atoms. The energy minimization was done till it reached a Root Mean Square Deviation (RMSD) cut-off of $0.18 \mathrm{~A}^{\circ}$ and the resulting structure was used for docking.

\section{IFD protocol}

IFD of the prepared ligands with the prepared proteins was performed using Induced Fit Docking protocol of GLIDE v5.5 from Schrödinger Suite, 2009 (Sherman et al., 2006). Induced fit docking combines GLIDE and
Prime refinement modules. Prime accurately predicts the ligand binding modes and concomitant structural changes in the receptor. Systematic and Simulation methods are adopted by glide for searching poses and ligand flexibility. Incremental construction for searching is employed by the systematic method, with Glide score (G-score) being the empirical scoring function. In IFD, both the ligand and the receptor are flexible which enables to dock the ligand at the receptor's binding site to generate multiple poses of the receptor-ligand complex, each including unique structural conformations of the receptor to fit the ligand pose and ranks them by Glide score (G-score) to find the best structure of the docked complex. G-score takes into account a number of parameters like hydrogen bonds (H-bond), hydrophobic contacts (Lipo), van derWaals (vdW), columbic (Coul), polar interactions in the binding site (Site), metalbinding term (Metal) and penalty for buried polar group (BuryP) and freezing rotatable bonds (RotB). The calculation of GScore in $\mathrm{Kcal} / \mathrm{mol}$ is: G-Score $=\mathrm{H}$ bond + Lipo + Metal + Site + 0.130 Coul + 0.065 vdW - Bury P - RotB. Where Hbond $=$ Hydrogen bonds, Lipo = hydrophobic interactions, Metal - metal binding term, Site $=$ Polar interactions in the binding site, $\mathrm{vdW}=$ Vander Waals forces, Coul = coulombic forces, Bury $\mathrm{P}=$ penalty for buried polar group, $\operatorname{Rot} B=$ freezinf rotatable bonds. The prepared protein was docked with the minimized ligands. The active sites in the protein 6COX_A were selected to be docked with the ligand. The prepared structure of 6COX_A was used for induced fit docking simulations. IFD was performed and best conforma-tions were selected based on Docking score, Glide energy, and Glide emodel scores. In IFD, both the ligand and the receptor are flexible which enables to dock the ligand at the receptor's binding site to generate multiple poses of the receptor-ligand complex, each including unique structural conformations of the receptor to fit the ligand pose and ranks them by docking score (D-score) to find the best structure of the docked complex. Initially a receptor grid, where the ligand has to be docked with the receptor was set by picking the centroid of the cocrystallized inhibitor (6COX) present at the active site. It creates a grid box and the size of the grid box was limited to $20 \mathrm{~A}^{\circ}$. The generation of different conformations of the docked complexes (poses) was set to a maximum of 20 . The poses generated were ranked based on D-score. The pose that made the maximum hydrogen bond (H-bond) interactions from phytocompounds docked complexes were considered for further analysis and the results are compared.

\section{Visualization and analysis}

The PyMol Molecular Graphics System (Kini and Evans., 1987) was used to analyze the protein structure, the hydrogen bond interactions with the active site residues and preparation of high resolution images. The 


\section{Table I}

\section{List phytocompounds in methanolic extract of Vitex negundo}

\begin{tabular}{|cllcc|}
\hline Compound & Phytocompound name & Molecular formula & Molecular weight & RT \\
\hline 1 & $\begin{array}{l}\text { 1-acetyl-2-(methylsulfonyl)methylindol-5-carbonsaure- } \\
\text { methylester }\end{array}$ & $\mathrm{C}_{14} \mathrm{H}_{15} \mathrm{NO}_{5} \mathrm{~S}$ & 309 & 36.1 \\
& 5-(N',N'-Dimethylthioureido)salicylic acid & $\mathrm{C}_{10} \mathrm{H}_{12} \mathrm{~N}_{2} \mathrm{O}_{3} \mathrm{~S}$ & 240 & 32.1 \\
3 & N,N'-Bis(2-aminophenyl)cyclohexane-1,2-diamine & $\mathrm{C}_{18} \mathrm{H}_{24} \mathrm{~N}_{4}$ & 296 & 27.6 \\
& $\begin{array}{l}\text { 3-(1-Acetylamino-1-cyclohexylmethyl)-1- } \\
\text { methylquinolin-2(1H)-one }\end{array}$ & $\mathrm{C}_{19} \mathrm{H}_{24} \mathrm{~N}_{2} \mathrm{O}_{2}$ & 312 & 36.1 \\
\hline
\end{tabular}

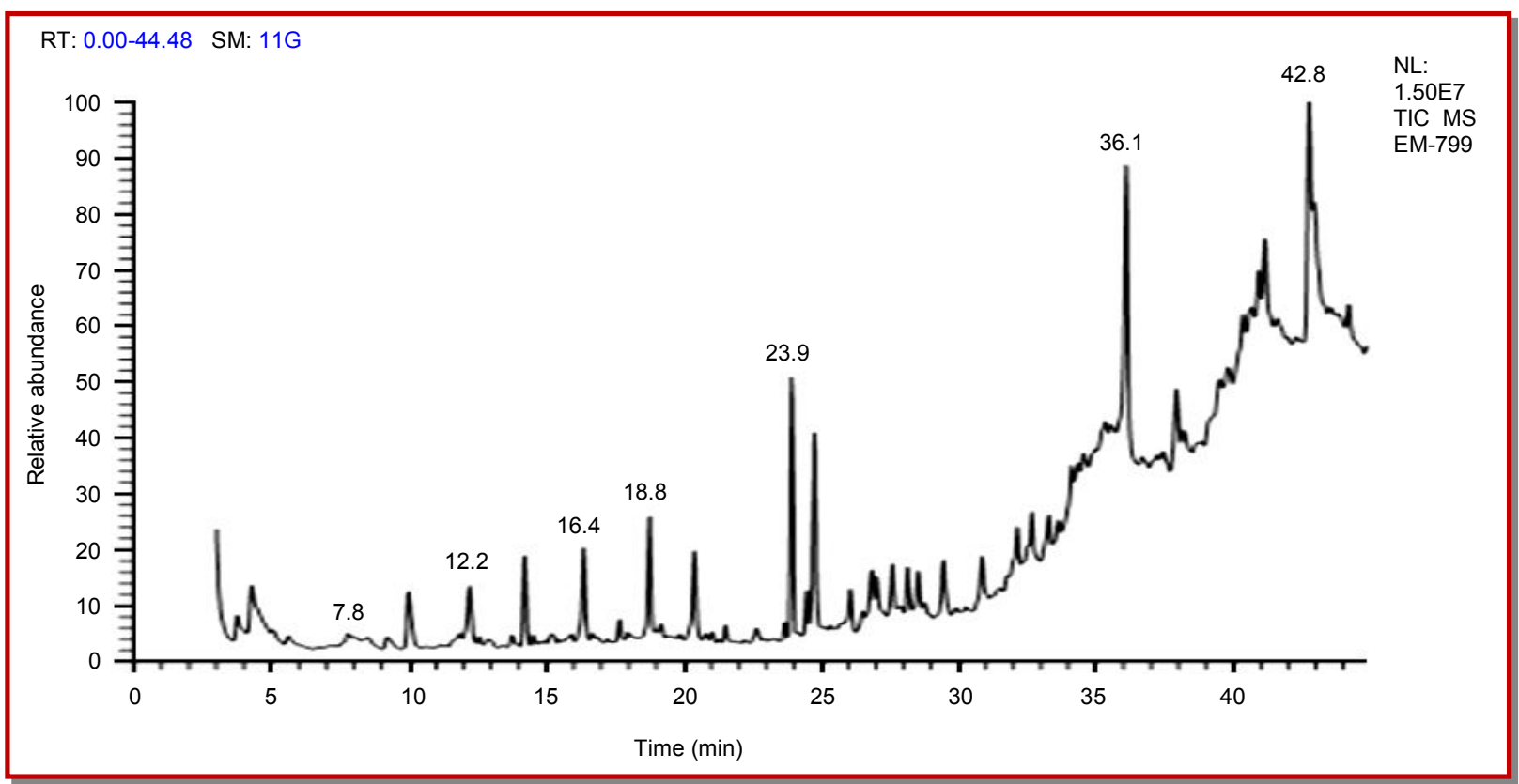

Figure 1: GCMS result of methanolic extract of Vitex negundo

hydrophobic interactions were obtained as Ligplot diagram by submitting the docked complexes to the online PDBsum server (http://www.ebi.ac.uk/ pdbsum).

\section{Result and Discussion}

Medicinal plants consist of several bioactive phytocompounds. Phytocompounds are used to treat various ailments. GCMS was performed for identification of phytocompounds. The methanolic leaf extract of $V$. negundo was taken for GCMS study. The GCMS method determined that the methanolic extract of $V$. negundo showed the presence of phenolic compounds (Table I). There are several phytocompounds present in methanolic leaf extract which are responsible for the antiinflammatory activity. The activities were identified by using PASS server. The structure conformation of the identified phytocompounds was accomplished by comparing the mass spectra obtained to their commercial Wiley9 library. The peaks indicate the presence of phytochemical constituents (Figure 1). GCMS result concludes that the phenolic compounds were present in the methanolic leaf extract of $V$. negundo.

\section{COX_A protein secondary structure prediction}

Cyclooxygenase (COX) enzyme converts arachidonic acid to prostaglandins, which have important signalling and housekeeping functions, particularly in platelets, the gastrointestinal tract, lungs, and kidneys (Mohanapriya et al., 2012; Adinarayana, 2012). COX-2 is an inducible isoforms that is found and expressed mainly in inflammatory and immune cells (Gogoi, 2012). COX-1 and COX-2 are $63 \%$ identical and $77 \%$ similar at the amino acid level. The result of COX-2 secondary structure prediction tool PSIPRED showed that the alpha helix is $36.5 \%$, wxtended strand is $13.6 \%$, beta turn is $3.9 \%$ and Random coil is $46 \%$ within the target 6COX_A sequence. Random coil was more than that of alpha helices. Beta turn is $3.9 \%$ lesser than the other secondary structures present in the sequence (Figure 2). Secondary structure prediction provides the 


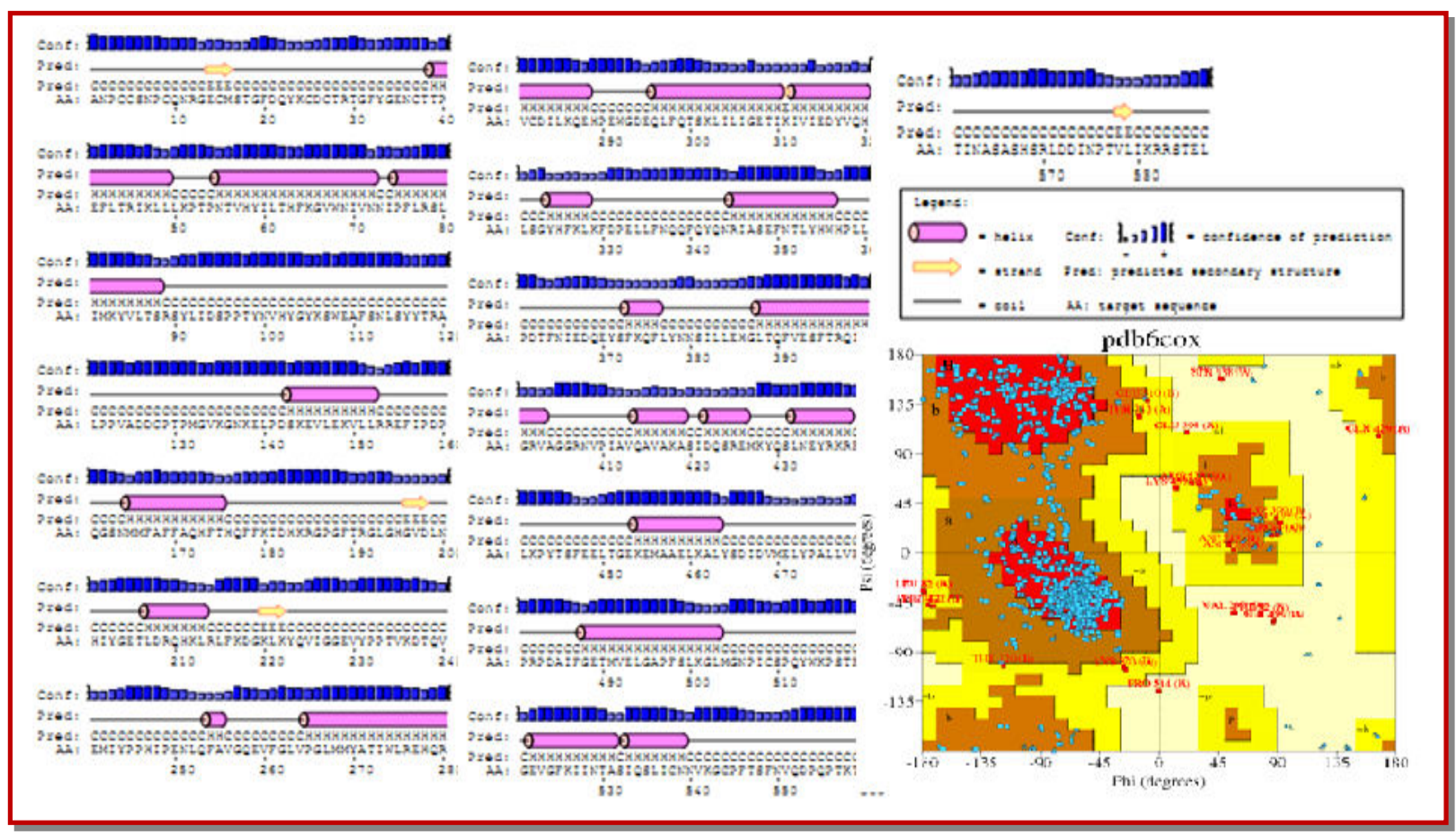

Figure 2: Secondary structure prediction of 6COX_A sequence using PSI-PRED server and Ramachandran plot for 6COX_A

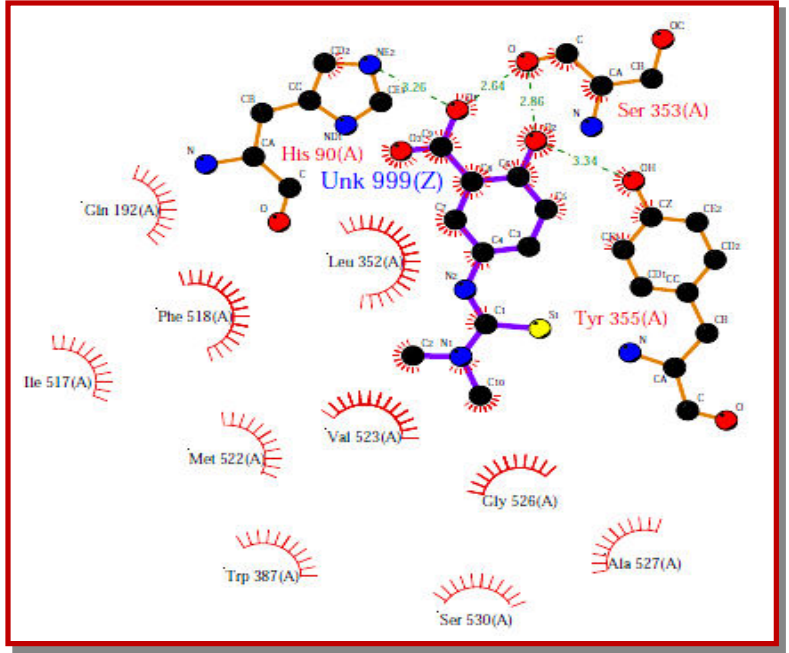

Figure 3: Protein-ligand interaction 2D map of 6COX_A with Compound_1 using Ligplot diagram

information about 6COX_A protein. ProQ is a neural network based predictor that predicts the quality of different parts of protein model based on the number of structural features. The ProQ results showed that 6COX_A protein model is a very good model.6COX_A protein secondary predicted LGscore is 2.3. Validation of the model 6COX_A protein by PROCHECK presented a Ramachandran plot analysis rendering $74.1 \%$ residues in the most favoured regions, $22.5 \%$ in the additionally allowed regions and 2.5\% Generously allowed regions (Figure 2). The binding pocket residues of the 6COX_A are His90, Arg120, Gln192, Val349, Leu352,
Ser353, Tyr355, Leu359, Tyr385, Arg513, Ala516, Phe518, Val523 and Ala527 respectively. These active sites of COX2 are able to binding with the ligands.

Molecular docking studies showed the potential of the phytocompounds of $V$. negundo against COX-2 enzyme. The phytocompounds bind to the active sites of the target protein, 6COX_A and have a good docking score and glide energy compared with the existing drugs such as aspirin and ibuprofen. The Induced Fit Docking simulation was performed by using GLIDE v5.5 from Schrödinger Suite 2009 with the phyto-compounds. The binding poses, interactions and distances were analyzed by Glide. The interactions of active sites were within $20 \mathrm{~A}^{\circ}$ distances. The interaction energy between the 6COX_A and phytocompound is computed as glide score and glide energy. The native ligand SC-558 is bounded in a hydrophobic cavity formed by GLN192, HIS90, SER353, ARG120, LEU352, TYR355, VAL523, MET522 residues and formed hydrogen interaction with the target protein.

All the compounds have high potential to bind with the active site. The glide score and glide energy for compound_1 were -9.7 and $-50.1 \mathrm{Kcal} / \mathrm{mol}$. The phytocompounds form hydrogen bonds with SER530, TRY355, HIS90 and ARG120 residues of COX-2 (Figure 3).The compound_1 ligand exhibited two hydrogen bond interactions in pose1(SER530[O-H....O], TYR355[O $-\mathrm{H} . . . \mathrm{O}])$ and in pose2 the ligand exhibited two intactions, (HIE90 [N-H....O] and ARG120[N-H....O]). The compound_1 1-acetyl-2-(methylsulfonyl) ethylindol -5-carbonsaureme-thylester shows the highest glide 


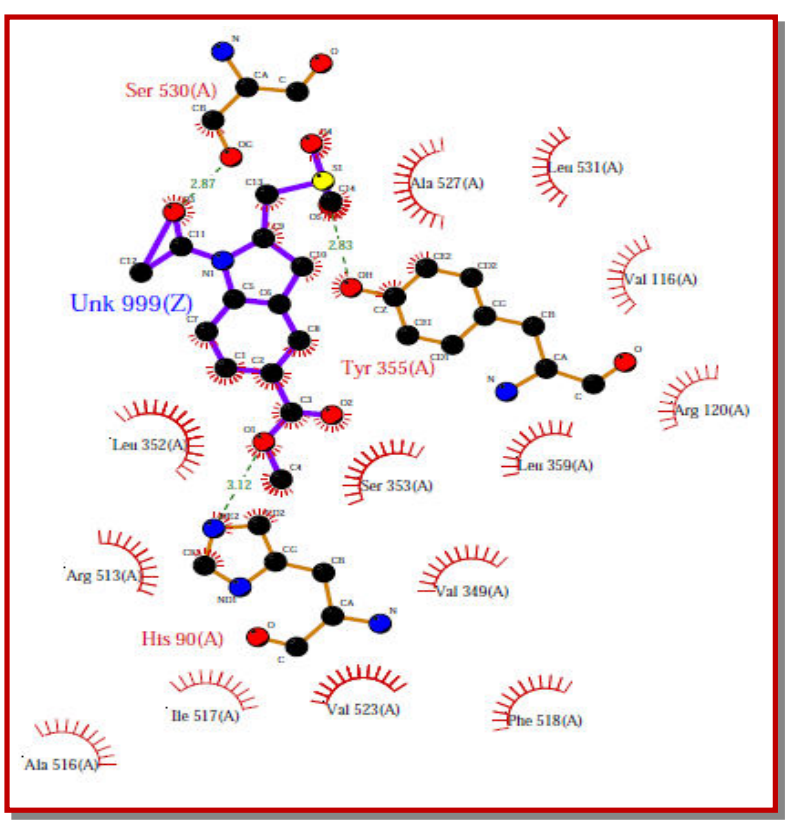

Figure 4: Protein-ligand interaction 2D map of 6COX_A with Compound_2 using Ligplot diagram

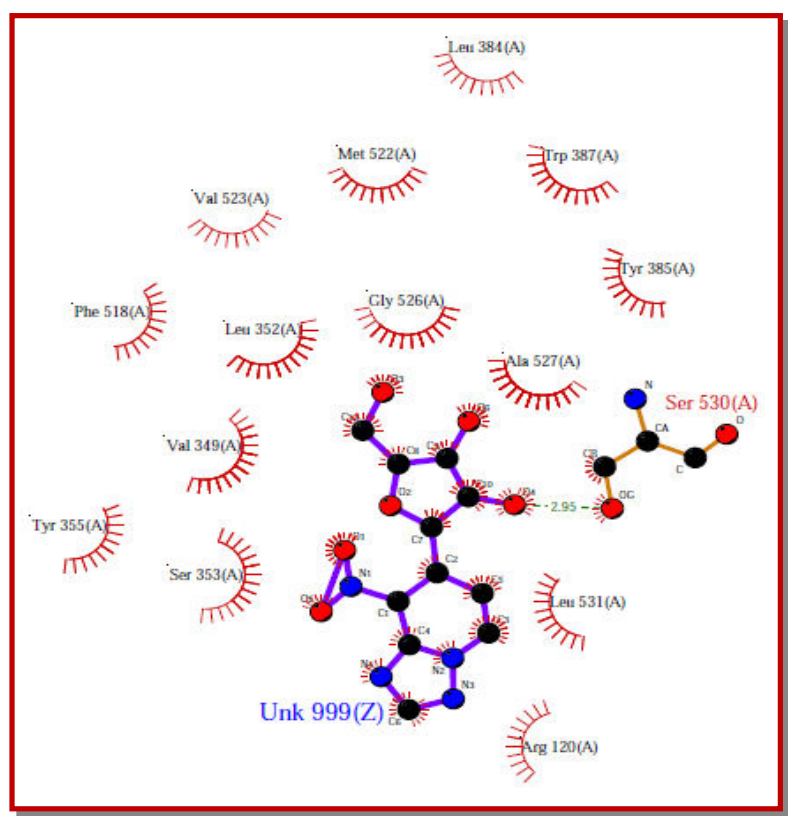

Figure 5: Protein-ligand interaction 2D map of 6COX_A with Compound_3 using Ligplot diagram

score than other three compounds and existing COX2 inhibitor such as aspirin and ibuprofen. The glide score and glide energy for compound_2 were -7.6 and -44.4 $\mathrm{Kcal} / \mathrm{mol}$. The compound _2 5-(N', N'- dimethylthioureido) salicylic acid showed hydrogen bond interaction with SER353, TRY355, and HIS90 residues. This compound when bound to the protein exhibited two hydrogen bond interactions ([O-H.... ] SER353 and [O-H.... $\mathrm{O}$ SER353). In pose2 the ligand formed two interactions (HIE90 [N-H.... ] and TYR355 [O-H....O])

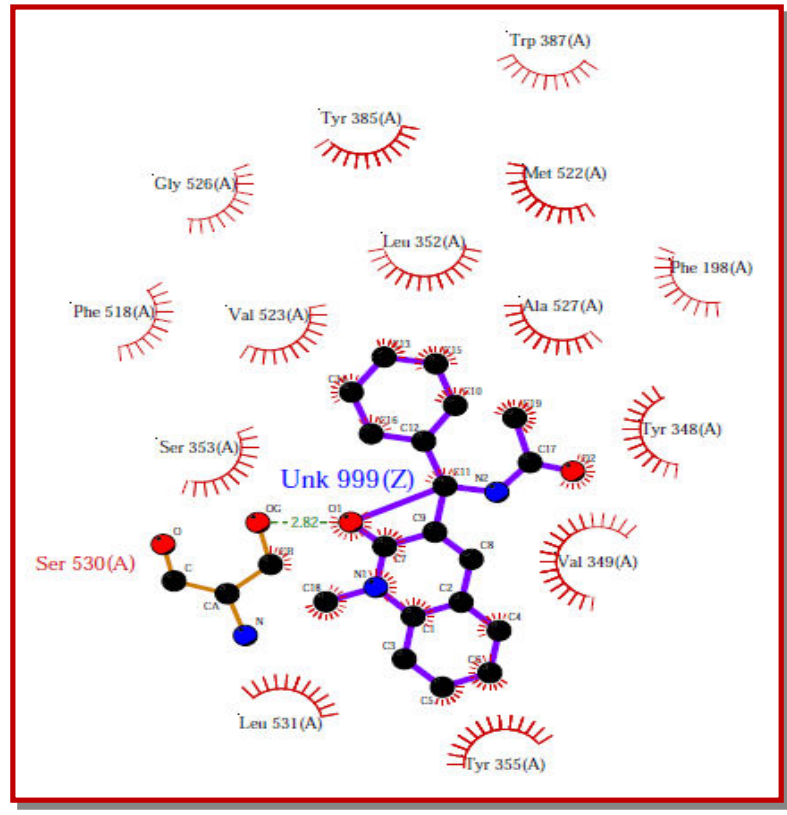

Figure 6: Protein-ligand interaction 2D map of 6COX_A with Compound_4 using Ligplot diagram

(Figure 4). The glide score and glide energy for compound_3 N,N'-Bis(2-aminophenyl)cyclohexane-1,2diamine were -8.8 and $-49.3 \mathrm{Kcal} / \mathrm{mol}$. The residues VAL523, MET522 were involved in hydrogen bond formation with compound_3. The compound exhibited two hydrogen bond interactions ([N-H....O] VAL523 and [O-H.... ] MET522) (Figure 5). The glide score and glide energy for compound_4 3-(1-Acetylamino-1cyclohexyl-methyl)-1-methylquinolin-2(1H)-one were 11.1 and $-50.0 \mathrm{Kcal} / \mathrm{mol}$. SER530, TRY355, and ARG120 were the protein residues involved in the hydrogen bond formation with the ligand. One hydrogen bond interaction was seen in the first pose (SER530 [OH.... ]) (Figure 6). The phyto-compounds show a better glide score and glide energy compared with COX2 inhibitors such as aspirin and ibuprofen. (Table II and III).

\section{Conclusion}

The present study showed that four phyto-chemical compounds could be the potential inhibitory source against 6COX_A protein. The phytocompounds of $V$. negundo showed a better docking simulation and interaction analysis, than the existing COX-2 inhibitor such as aspirin and ibuprofen.

\section{Acknowledgement}

The authors are grateful to Dr. D. Velmurugan, Bioinformatics Infrastructure Facility (DBT-BIF), University of Madras for providing computational lab facilities. 
Table II

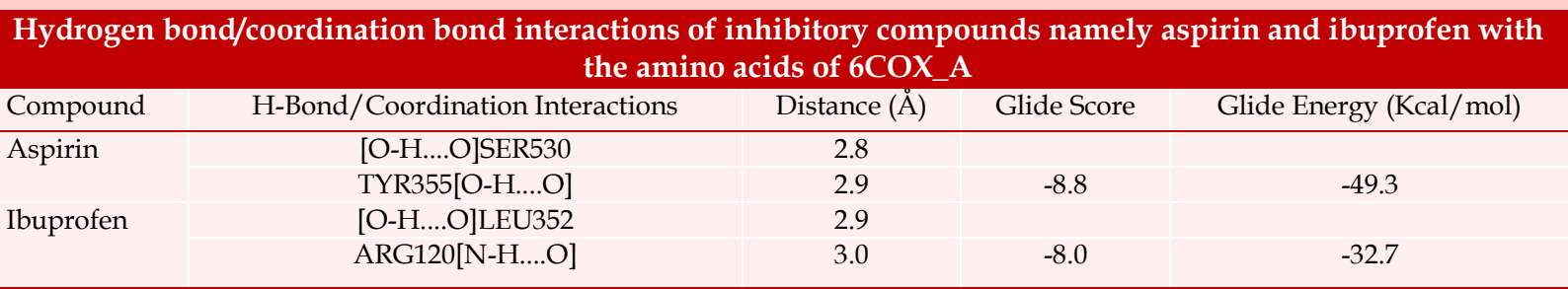

Table III

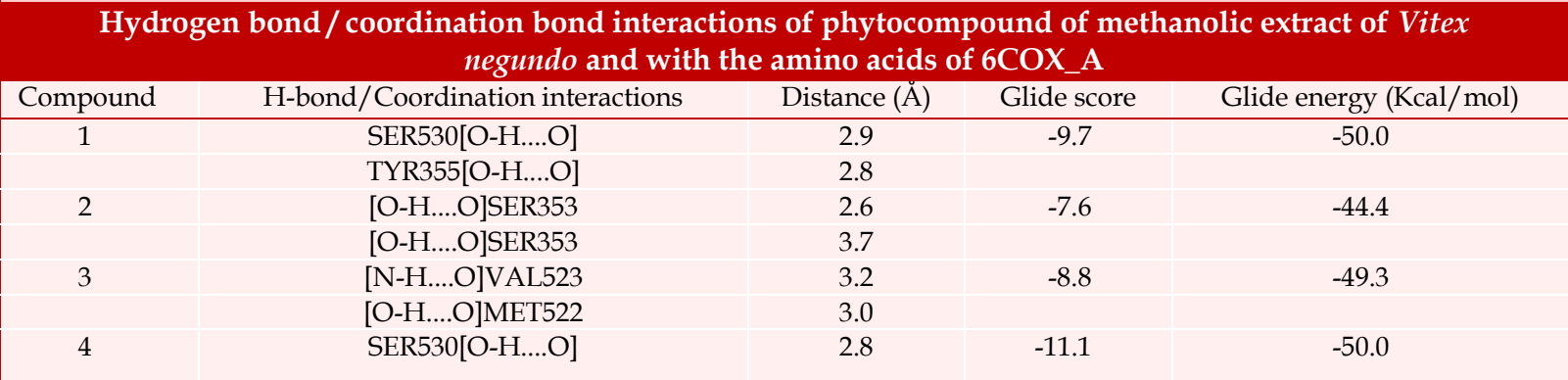

\section{References}

Adinarayana KPS, Reddy PA, Babu PA. Structural studies on docking COX-2 inhibitors. J Bioinformatics Res. 2012; 1: 2126.

Aswar PB, Khadabadi SS, Kuchekar BS, Rajurkar RM, Saboo1 $\mathrm{SS}$, Javarkar RD. In vitro evaluation of anti-bacterial and anti -fungal activity of Vitexnigundo (Verbenaceae). Ethno Leaf. 2009; 13: 962-67.

Bryson K, McGuffin LJ, Marsden RL, Ward JJ, Sodhi JS, Jones DT. Protein structure prediction servers at University College London. Nucleic Acids Res. 2005; 33: 36-38.

Castro JA, Sasame HA, Sussman H, Gillette JR. Diverse effects of SKF 525-A and anti-oxidants on carbon tetrachlorideinduced changes in liver microsomal P-450 content and ethylmorphine metabolism. Life Sci. 1968; 7: 129-36.

Dharmasiri MG, Jayakody JRAC, Galhena G, Liyanage SSP, Ratnasooriya WD. Anti-inflammatory and analgesic activities of mature fresh leaves of Vitex negundo. J Ethnopharmacol. 2003; 87: 199-206.

Dundas J, Ouyang Z, Tseng J, Binkowski A, Turpaz Y, Liang. CASTp: Computed atlas of surface topography of proteins with structural and topographical mapping of functionally annotated residues. Nucleic Acids Res J. 2006; 34: 116-18.

Ghosh A. Herbal folk remedies of Bankura and Medinipur districts, West Bengal (India). Indian J Tradit Knowl. 2003; 2: 393-96.

Gierse JK, McDonald JJ, Hauser SD, Rangwala SH, Koboldt $\mathrm{CM}$, Seibert K. A single amino acid difference between cyclo -oxygenase-1 (Cox-1) and -2 (Cox-2) reverses the selectivity of Cox-2 specific inhibitors. J Biol Chem. 1996; 271: 15810-14.

Gogoi D, Bezbaruah RL, Bordoloi M, Sarmah R, Bora TC. Insights from the docking analysis of biologically active compounds from plant Litsea Genus as potential COX-2 inhibitors. Bioinformation 2012; 8: 812-15.

Jones DT. Protein secondary structure prediction based on position-specific scoring matrices. J Mol Biol. 1999; 292: 195202.

Khokra S, Prakash O, Jain S, Aneja K, Dhingra Y. Essential oil composition and antibacterial studies of Vitex negundo Linn. extracts. Indian J Pharm Sci. 2008; 70: 522-26.

Kini RM, Evans HJ. Structure-function relationships of phospholipases. The anticoagulant region of phospholipases A2. J Biol Chem. 1987; 262: 14402-07.

Kulkarni PR, Virkar AD, D'Mello P. Anti-oxidant and antiinflammatory activity of Vitex negundo. Indian J Pharm Sci. 2008; 70: 838-40.

Kurumbail RG, Stevens AM, Gierse JK, McDonald JJ, Stegeman RA, Pak JY, Gildehaus D, Miyashiro JM, Penning TD, Seibert K, Isakson PC, Stallings WC. Structural basis for selective inhibition of cyclooxygenase-2 by anti-inflammatory agents. Nature 1996; 384: 644-48.

Laskowski RA, MacArthur MW, Moss DS, Thornton JM. PROCHECK: A program to check the stereochemical quality of protein structures. J Appl Cryst. 1993; 26: 283-91.

Laskowski RA, Swindells MB. LigPlot+: Multiple ligandprotein interaction diagrams for drug discovery. J Chem Inf Model. 2011; 51; 2778-86.

Mahmud S, Shareef H, Farrukh U, Kamil A, Rizwani GH. Antifungal activities of Vitex negundo linn. Pak J Bot. 2009; 41: 1941-43.

Mohanapriya A, Achuthan D. Comparative QSAR analysis of cyclooxygenase-2 inhibiting drugs. Bioinformation 2012; 8: 353-58.

Nathan C. Points of control in inflammation. Nature 2002; 420: 


\section{$846-52$.}

Raji R. Biological activities, chemical constituents and medicinal value of Vitex negundo Linn. Universal J Pharm. 2013; 2: 42-46.

Saqib A, Karigar CS. Cyclooxygenase isoforms in health and disease. Internet J Pharmacol. 2009; 7: 1.

Sherman W, Day T, Jacobson MP, Friesner RA, Farid R. Novel procedure for modeling ligand/receptor induced fit effects. J Med Chem. 2006; 49: 534.

Sudha KN, Shakira M, Prasanthi P, Sarika N, Kumar N, Babu PA. Virtual screening for novel COX-2 inhibitors using the ZINC database. Bioinformation. 2008; 2: 325.
Tandon VR. Medicinal uses and biological activities of Vitex negundo. Nat Prod Radiance. 2005; 4: 162-65.

Telang RS, Chatterjee S, Varshneya C. Studies on analgesic and anti-inflammatory activities of Vitex negundo Linn. Indian J Pharmacol. 1999; 31: 363-66.

Tiwari OP, Tripathi YB. Anti-oxidant properties of different fractions of Vitex negundo Linn. Food Chem. 2007; 100: 117076.

Vishwanathan AS, Basavaraju R. A review on Vitex negundo L.: A medicinally important plant. Eur J Biol Sci. 2010; 3: 30-42.

Wallner B, Elofsson A. Can correct protein models be identified? Protein Sci. 2003; 12: 1073-86.

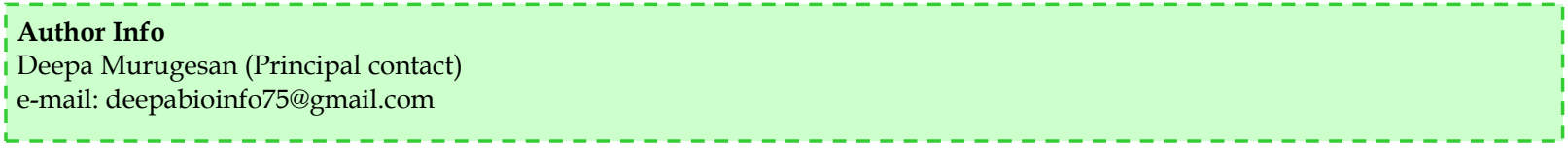

